The reversal of the transamination to pyruvate has also been demonstrated. Incubation of washed ratliver particles with $\mathrm{L}$-alanine and $\alpha$-keto- $n$-butyrate led to the formation of $\alpha$-amino- $n$-butyric acid, while with $\alpha$-ketoisocaproate they gave leucine, and with phenylpyruvate they gave phenylalanine. (In these experiments the final concentration of $\mathrm{L}$-alanine was $0.03 \mathrm{M}$; that of the keto-acid was $0.025 M$. The incubation conditions and subsequent operations were the same in these experiments, and in all the experiments to be described, as those detailed above.) These syntheses were not effected when $D$-alanine or L-aspartate was substituted for L-alanine.

Other animal tissue preparations that have been examined, using the same experimental techniques, for transamination to pyruvate and $\alpha$-ketoglutarate, are listed in the accompanying table; + denotes their ability, - their inability, to catalyse transamination with the substrates indicated. The whole range of amino-acids tested with each preparation is noted; it may be that other amino-acids would behave differently.

Experiments with dialysed supernatants of ratliver homogenates show that they are able to catalyse transamination from L-phenylalanine, L-methionine and $\mathrm{L}$-leucine, directly to $\alpha$-keto- $n$-butyrate to produce $\alpha$-amino-n-butyric acid (Fig. 3), from L-leucine to phenylpyruvate to give phenylalanine, and from L-phenylalanine to $\alpha$-ketoisocaproate to give leucine. In these experiments the $\mathrm{x}$-amino-acids were at a final concentration of $0.03 M$ and the $\alpha$-keto acids $0.025 M$. (Sodium $\alpha$-ketoisocaproate and $\alpha$-keto- $n$-butyrate were kindly provided by Dr. A. Meister.)

It is evident that biological transamination includes in its scope a much wider range of $\alpha$-keto-acid acceptors than has hitherto been recognized. It is also obvious, even from the application of this qualitative method for the analysis of amino-acids, that big differences exist between different animal species and tissues in their transaminating ability. Many potential amino-group donors and keto-acid acceptors, including peptides and ketopeptides, will need to be investigated for their interaction in a wide range of organisms and tissues before the full scope of the transamination reaction can be appreciated.

I wish to thank Dr. D. M. Needham and Dr. F. J. R. Hird for their advice and encouragement during the course of this work, and to acknowledge a grant from the Medical Research Council.

${ }^{1}$ Cammarata, P. S., and Cohen, P. P., J. Biol. Chem., 187. 439 (1950). ${ }^{2}$ Feldman, I. I., and Gunsalus, I. C., J. Biol. Chem., 187, 821 (1950).

${ }^{3}$ Hird, F. J. R., and Rowsell, E. V., Nature, 168, 517 (1950).

“Braunstein, A. E., “Adv. Protein Chem.”, 3, 1 (1947).

\section{ULTRASONICS IN FLUIDS}

TNDER the auspices of the Royal Flemish Academy and the United Nations Educational, Scientific and Cultural Organization, an international conference on ultrasonics was held in Brussels during June 7-9 under the presidency of Prof. A. van Itterbeek; the secretary was Prof. A. J. Rutgers. There were about one hundred participants representing most countries of Western Europe, the United States of America and Australia. It may indeed seem that workers in ultrasonics are insatiable in discussing their activities, since this congress followed so closely on the heels of those of Erlangen (1949) and Rome (1950); but the earlier meetings dealt rather with applications of ultrasonics, whereas this year's meeting was concerned entirely with physical measurements and theories.

The subjects proposed for discussion were the following : (a) absorption and dispersion in gases and in pure liquids ; developments in the field of "second viscosity of liquids'; $(b)$ absorption in electrolytic solutions; relaxation time for ionic interactions; ultrasonic vibration potentials in colloidal solutions; (c) absorption in liquid mixtures or in liquids containing solid particles or gas bubbles ; $(d)$ absorption in liquid helium; 'second sound'.

On the first morning, a lively discussion soon developed on the significance of the 'streaming' of fluid from the surface of an ultrasonic emitter contained in a tube, and the vortices so set up, from the point of view of the dilatational viscosity which Stokes invoked only to ignore. It was pointed out that similar circulations can be set up in a liquid at quite low frequencies, so that it may be difficult to distinguish any specific effect at high frequencies, when the 'second viscosity' would be expected to show itself. This leads to alternative explanations of the phenomenon, based on the equations of compressible and incompressible flow, respectively. Yet a third theory was adduced in the first category, in which the streaming due to the breakdown of shock wave-fronts was discussed.

A large proportion of the papers presented to the congress were concerned with relaxation phenomena in gases and liquids. On the theoretical side, much argument took place on the best way of representing anomalous absorption, whether by a complex coefficient of viscosity or by single or multiple relaxation times, usually of thermal type.

For water near $4^{\circ} \mathrm{C}$, a configurational relaxation between two types of structure having different compressibilities seems best to represent the observed absorption/temperature curve. In applying these theories to actual fluids, with the possible exception of hydrogen, some data have to be taken from the experimental results and it is well, as one speaker reminded us, that we should be clear in our own minds when we are doing this.

On the experimental side, new results were presented for velocity and absorption in organic liquids and mixtures thereof, electrolyte solutions, liquid hydrogen and liquid helium. Besides the common variable-path interferometers and pulse techniques, som? data were presented from measurements with the radiation pressure, probe microphone, hot-wire, reverberation and Schlieren methods. Interesting reports were also presented of experiments on scattering by solids or liquid bodies surrounded by other liquids, and of the setting-up of potential fields in colloids under the action of ultrasonics.

Measurements of ultrasonic absorption in the critical regions between gas and liquid on one hand and between solid and liquid on the other have recently been made. Glycerine in the neighbourhood of $-10^{\circ} \mathrm{C}$. exhibits the latter transition, showing a maximum of absorption to $30 \mathrm{Mc}$./sec. waves at this temperature. Some theories relating to relaxation in this quasi-solid state were advanced, though it is interesting to note that the existence of relaxation frequencies in solids was first discussed by Prandtl for the sonic range in 1928. The ultrasonic relaxations for liquids discovered later bear a close resemblance, in a different part of the gamut, to these.

The final morning was devoted to the propagation of sound in liquid helium II, wherein both acoustic 
and thermal waves ('first' and 'second' sound) may be propagated. The interactions of these two types and the effect of a temperature boundary-layer which forms at the walls of the containing vessel were discussed from the theoretical point of view by a number of speakers, while experiments were adduced relating to the discontinuity in velocity of 'first' sound at the lambda-point and the theoretical relation which would make this velocity equal to three times the velocity of 'second' sound as absolute zero is approached.

The papers (about thirty-five in all) presented to the conference will eventually be published (in English, French or German) in the Mededelingen Koninklijke Vlaamsche Academie voor Wetenschappen (Brussels). It is hoped that summaries of many of the papers will appear in a fortheoming number of the journal Acustica.

E. G. RICHARDSON

\section{CATION EXCHANGE AND CLAY MINERAL STRUCTURE}

A MEETING of the Clay Minerals Group of the A Mineralogical Society, held at Rothamsted Experimental Station, Harpenden, during April 27-28, was largely devoted to a discussion on cation exchange and clay mineral structure, although some general papers were also read and discussed. On the evening of April 27 a lecture on the development of the fuller's earth industry, and the uses of fuller's earth from earliest times, was delivered by R. H. S. Robertson (Glasgow).

The discussion on cation exchange and clay mineral structure was introduced by Dr. D. M. C. MacEwan (Rothamsted), who, with the aid of models, outlined the structures proposed for montmorillonite by Hofmann, Endell and Wilm ${ }^{1}$, and by Edelman and Favejer ${ }^{2}$, and pointed out that this discussion was, in effect, an attempt to see how far the available data could help towards a decision as to the most probable structure.

Prof. H. Deuel (Zurich) described the preparation and properties of organo-silicon compounds, especially the silanols containing $\mathrm{Si}-\mathrm{OH}$ groups, and pointed out that Edelman and Favejee have postulated such groups in clay minerals. The previous work of Berger ${ }^{3}$, and others, has shown that clays can give organic derivatives, and Prof. Deuel himself has prepared a considerable range of such compounds using 1,2-epoxides, mustard gas, etc. Thionyl chloride gives extremely reactive clay chlorides, which react with, for example, alcohol to give esters, etc. The decrease in cation-exchange capacity after esterification corresponds exactly to the amount of ester groups introduced, and the original cation-exchange capacity returns on hydrolysis. Similar esters prepared from different clay minerals have different properties, an observation which may lead to the development of a method for separation of minerals. In the discussion, H. R. Samson (Rothamsted) suggested that the reactions might be due partly to the hydroxyl groups of decomposition products formed by reaction of the clay with drastic reagents such as thionyl chloride, or of $\mathrm{Al}(\mathrm{OH})^{++}$, present in the hydrogen-montmorillonite. Dr. B. S. Neumann (Fuller's Earth Union, Ltd.) inquired whether free silica gel in the samples might not cause such reactions.

Recent attempts to distinguish between the two structures were described by M. J. Méring (Paris), who pointed out that, on the Hofmann-Endell-Wilm theory, structural water should amount to $5 \cdot 1$ per cent, while on the Edelman-Favejee one it should be $6 \cdot 1$ per cent. With normal analytical methods, it is difficult to distinguish between the final removal of sorbed moisture and the beginning of removal of structural water. However, Mile. Glaeser's use of the Fischer reagent for determination of sorbed water seems promising. Surface areas of calcium- and sodium-montmorillonites with varying water contents and $\mathrm{X}$-ray data for calcium-sodium-montmorillonites, etc., have also been examined. The results tend to favour the Hofmann-Endell-Wilm structure rather than the Edelman-Favejee one. In the discussion, P. Franzen (Amsterdam) suggested that application of the methods of $M$. Méring to the compounds of Prof. Deuel may lead to considerable further information.

The observation that lithium-montmorillonite loses water irreversibly at a much lower temperature (about $150^{\circ}$ C.) than montmorillonite saturated with other cations was discussed by R. Greene-Kelly (Rothamsted). Méring's suggestion that the formation of $\mathrm{O}-\mathrm{Ca}-\mathrm{O}$ bonds ${ }^{4}$ between the sheets in calciummontmorillonite causes the sheets to be locked in position and therefore irreversibly dehydrated can scarcely hold for $\mathrm{Li}^{+}$, although it might be that $\mathrm{Li}^{+}$ would react with one of the hydroxyl groups. Attention was directed to other peculiarities of lithium-montmorillonite $\theta^{5}$ and lithium-vermiculite $\theta^{6}$, in the ensuing discussion, by Dr. R. C. Mackenzie and Miss A. A. Milne (both of Aberdeen). M. Méring stated that he has now abandoned the $\mathrm{O}-\mathrm{Ca}_{\mathrm{C}} \mathrm{O}$ - hypo. thesis, while Prof. Deuel suggested that $\mathrm{Si}-\mathrm{O}-\mathrm{Si}$ links might be formed by condensation of two $\mathrm{Si}-\mathrm{OH}$ groups in separate layers. $\mathrm{He}$ also suggested the study of irreversible dehydration in hydrogenmontmorillonite; but several speakers doubted the existence of this material.

Prof. D. McConnell (Ohio) communicated a paper on the calculation of the structural formula of montmorillonite, assuming three cations (silicon, hydrogen and aluminium) present in the tetrahedral layer, that is, partial substitution of $\left(\mathrm{OH}_{4}\right.$ for $\mathrm{SiO}_{4}$ ?. Some doubt was cast on the existence of tetrahedral hydroxyl groups by R. Greene-Kelly, who showed some thermograms for one sample of montmorillonite saturated with various cations, where the relative sizes of the two high-temperature endothermic peaks vary with the exchangeable cation. K. Norrish (Rothamsted) mentioned that Hendricks has sug. gested that the final endothermic peak may be due to isolated hydroxyl groups unable to condense until the final recrystallization takes place-a suggestion supported by Dr. S. J. Gregg (Exeter), who pointed out the difficulty in removing the last traces of a gas in many decompositions, for example, calcium carbonate, magnesium carbonate, etc. The possibility that hydroxyl groups in McConnell's structure would be partially dissociated and give rise to cationexchange was suggested by Prof. Deuel.

Experiments on the effect of grinding on micas, described by Miss Milne and Dr. Mackenzie, showed that the cation-exchange capacity of muscovite increases to about $130 \mathrm{~m}$.equiv. $/ 100 \mathrm{gm}$. after nine hours of grinding, remains constant at this value for about another nine hours and then tends to increase again. Corresponding changes observed in the X-ray data indicate almost complete breakdown of the structure by nine hours, with gradual recrystallization into illite thereafter. The cation-exchange capacity 